\title{
Gate-induced superconductivity in a monolayer topological insulator
}

\author{
Ebrahim Sajadi', Tauno Palomaki², Zaiyao Fei², Wenjin Zhao², Philip Bement', Christian Olsen', \\ Silvia Luescher ${ }^{1}$, Xiaodong $\mathrm{Xu}^{2,3}$, Joshua A. Folk ${ }^{1 *}$, David H. Cobden ${ }^{2 *}$
}

\begin{abstract}
${ }^{1}$ Stewart Blusson Quantum Matter Institute, University of British Columbia, Vancouver, BC V6T 1Z4, Canada, and Department of Physics and Astronomy, University of British Columbia, Vancouver, BC V6T 1Z1, Canada. 'Department of Physics, University of Washington, Seattle, WA 98195, USA. ${ }^{3}$ Department of Materials Science and Engineering, University of Washington, Seattle, WA 98195, USA.
\end{abstract}

${ }^{*}$ Corresponding author. Email: cobden@uw.edu (D.H.C.); jfolk@physics.ubc.ca (J.A.F.)

The layered semimetal $\mathrm{WTe}_{2}$ has recently been found to be a two-dimensional topological insulator (2D TI) when thinned down to a single monolayer, with conducting helical edge channels. We report here that intrinsic superconductivity can be induced in this monolayer 2D TI by mild electrostatic doping, at temperatures below $1 \mathrm{~K}$. The 2D TI-superconductor transition can be easily driven by applying a small gate voltage. This discovery offers possibilities for gate-controlled devices combining superconductivity and non-trivial topological properties, and could provide a basis for quantum information schemes based on topological protection.

Many of the most important phenomena in condensed matter emerge from the quantum mechanics of electrons in a lattice. The periodic potential of the lattice gives rise to Bloch energy bands of independent fermions; on the more exotic side, electrons in a lattice can pair up into bosons and condense into a superconducting macroscopic quantum state, which conducts electricity with zero resistance. Relatively recently, it was realized that Bloch wavefunctions can have a non-trivial topology, leading to the discovery of topological insulators-materials that are electrically insulating in their interior but have conducting boundary modes (1). The first of these to be studied was the so-called 2D topological insulator (2D TI), in which one-dimensional helical edge modes (spin locked to momentum) give rise to the quantum spin Hall effect (2-4).

Materials that combine non-trivial topology with superconductivity have been the subject of active investigation in recent years (5-7). Here we report that monolayer $\mathrm{WTe}_{2}$, recently shown (8-13) to be an intrinsic $2 \mathrm{D} \mathrm{TI}$, turns superconducting under moderate electrostatic gating. Several other non-topological layered materials superconduct in the monolayer limit, either intrinsically or under heavy doping using ionic liquid gates (14-22). In monolayer $\mathrm{WTe}_{2}$, however, the phase transition to a superconducting state is from a 2D topological insulator, and occurs at such low carrier density that it can be readily induced by a simple electrostatic gate. The discovery may lead to gateable superconducting circuitry, and offers the potential to develop topological superconducting devices in a single material, as opposed to the hybrid constructions currently required (5).
We present data from two monolayer WTe ${ }_{2}$ devices, M1 and M2, with consistent superconducting characteristics. Each contains a monolayer flake of $\mathrm{WTe}_{2}$ encapsulated along with thin platinum electrical contacts between hexagonal boron nitride (hBN) dielectric layers. Figure 1A shows an image of M1, which has seven contacts along one edge, together with a side view and a schematic showing the configuration used to measure the linear 4-probe resistance, $R_{x x}$ $=d V / d I$. Top and bottom gates, at voltages $V_{\mathrm{t}}$ and $V_{\mathrm{b}}$ and with areal capacitances $c_{\mathrm{t}}$ and $c_{\mathrm{b}}$ respectively, can be used to induce negative or positive charge in the monolayer $\mathrm{WTe}_{2}$, producing an areal doping density given by $n_{e}=\left(c_{\mathrm{t}} V_{\mathrm{t}}+\right.$ $\left.c_{\mathrm{b}} V_{\mathrm{b}}\right) / e$, where $e$ is the electron charge. Note that we do not interpret this as a carrier density because the insulating state may be of correlated nature (as in, for example, an excitonic insulator); in addition, Hall density measurements are challenging owing to the 2D TI edge conduction. More details about gating, contact resistances and capacitances are given in (23).

Figure 1B illustrates the electrostatic tuning of M1 from p-doped conducting behavior at negative gate voltage, through an insulating state, to an n-doped highly conducting state at positive gate voltage. M1 is the same device whose insulating state was investigated in (9), and there demonstrated to be a 2D TI $(9,13)$; at $n_{e}=0, R_{x x}$ is more than $10^{7} \Omega$ owing to a meV-scale gap that blocks edge conduction below $1 \mathrm{~K}$ (see later in the text and (23)). For $n_{e}$ above $n_{\text {crit }} \approx+5 \times 10^{12} \mathrm{~cm}^{-2}$, however, the resistance drops dramatically when the sample is cooled, reaching the noise floor of the experiment (around $0.3 \Omega$ ) for $n_{e}>+7 \times 10^{12}$ 
$\mathrm{cm}^{-2}$ at $20 \mathrm{mK}$, indicating the appearance of superconductivity. Figure $1 \mathrm{C}$ is a phase diagram constructed from these and similar measurements discussed below. The emergence of a superconducting phase in direct proximity to a 2D TI phase, and at a doping level achievable with a single electrostatic gate, is the primary result of this paper.

The transition from an insulating to a metallic/superconducting $T$ dependence-the crossing of $R_{x x}$ lines in Fig. $1 \mathrm{~B}-$ occurs at $2.4 \mathrm{k} \Omega$. This corresponds to a square resistivity $\rho \approx 20 \mathrm{k} \Omega$, with a substantial uncertainty because the precise distribution of current in the device is not known (23). The evolution of the $T$ dependence with $n_{e}$ is illustrated in Fig. 2A. For all densities shown, the collapse of $R_{x x}$ with temperature is gradual, as expected for materials where the normal state 2D conductivity is not much greater than $e^{2} / h$. We define a characteristic temperature, $T_{1 / 2}$, at which $R_{x x}$ falls to half its $1 \mathrm{~K}$ value. Although this specific definition is somewhat arbitrary, it is typical in the literature $(15,21,22)$, and does not significantly affect any of our conclusions (23). Measured values of $T_{1 / 2}$ are shown as red dots on the phase diagram in Fig. $1 \mathrm{C}$ to indicate the boundary of superconducting behavior.

The superconductivity is suppressed by perpendicular $\left(B_{\perp}\right)$ or in-plane $\left(B_{\|}\right)$magnetic field (Fig. 2, B and C). For perpendicular field, orbital effects are expected to dominate (24-26). The dependence of $T_{1 / 2}$ on $B_{\perp}$ (Fig. 2B, inset) in the low-field limit is consistent with the linear $B_{c 2}^{\perp}(T)$ expected from Ginzburg-Landau theory. The characteristic perpendicular field in the low temperature limit is $B_{1 / 2}^{\perp}(T \rightarrow 0) \approx 25 \mathrm{mT}$, based on the measurements in Fig. 2B (inset). ( $B_{1 / 2}^{\perp}$ is the magnetic field where $R_{x x}$ falls to half its normal state resistance.) Estimates for the superconducting coherence length can be obtained either from the slope of $B_{1 / 2}^{\perp}(T)$ near $T_{1 / 2}$ or from $B_{1 / 2}^{\perp}(T \rightarrow 0)$, yielding $\xi_{\text {meas }}=100$ $\pm 30 \mathrm{~nm}$ in both cases (23).

The fact that $\xi_{\text {meas }}$ is significantly larger than the estimated mean free path $\lambda=h /\left(e^{2} \rho \sqrt{g_{\mathrm{s}} g_{\mathrm{v}} \pi n_{\mathrm{e}}}\right) \approx 8 \mathrm{~nm}$ suggests that the system is in the dirty limit $(\lambda<<\xi)$. Here $e$ is the electron charge and $h$ is the Planck constant. To calculate $\lambda$ we use spin and valley degeneracies $g_{\mathrm{s}}=g_{\mathrm{v}}=2$, and density and normal-state resistivity reflecting the conditions for Fig. $2 \mathrm{~B}, n_{e}=20 \times 10^{12} \mathrm{~cm}^{-2}$ and $\rho \approx 2 \mathrm{k} \Omega$ respectively. The coherence length expected in the dirty limit is $\xi \approx \sqrt{\hbar D / \Delta_{0}}$, for zero-temperature gap $\Delta_{0}=1.76 k_{\mathrm{B}} T_{\mathrm{c}}$ and diffusion constant $D$, where $\hbar$ is the reduced Planck constant and $k_{\mathrm{B}}$ is the Boltzmann constant. Indeed, using $T_{1 / 2}=$ $700 \mathrm{mK}$ for $T_{\mathrm{c}}$, and $D=2 \pi \hbar^{2} / g_{\mathrm{s}} \mathrm{g}_{\mathrm{v}} m^{*} e^{2} \rho \approx 12 \mathrm{~cm}^{2} \mathrm{~s}^{-1}$ (from the Einstein relation) with effective mass $(27) m^{*}=0.3 m_{e}$ gives $\xi \approx 90 \mathrm{~nm}$, where $m_{e}$ is the electron mass, consistent with $\xi_{\text {meas. }}$

For in-plane field, the atomic thinness of the monolayer makes orbital effects small. In the absence of spin scattering, superconductivity is then suppressed when the energy associated with Pauli paramagnetism in the normal state overcomes the superconducting condensation energy. This is referred to as the Pauli (Chandrasekar-Clogston) limit (28), and gives a critical field $B_{\mathrm{P}}=1.76 k_{\mathrm{B}} T_{\mathrm{c}} / g^{1 / 2} \mu_{\mathrm{B}}$, where $k_{\mathrm{B}}$ is the Boltzmann constant and $\mu_{\mathrm{B}}$ is the Bohr magneton. Assuming an electron $g$-factor of $g=2$ and taking $T_{\mathrm{c}}=700 \mathrm{mK}$ gives $B_{\mathrm{P}} \approx 1.3 \mathrm{~T}$. However, the data in Fig. $2, \mathrm{C}$ and F, indicate superconductivity persisting to $B_{1 / 2}^{\|}=3 \mathrm{~T}$.

Similar examples of $B_{1 / 2}^{\|}$exceeding $B_{\mathrm{P}}$ have recently been reported in other monolayer dichalcogenides, $\mathrm{MoS}_{2}$ and $\mathrm{NbSe}_{2}$, but the Ising superconductivity mechanism (15, 21) invoked in those works cannot explain an enhancement of $B_{1 / 2}^{\|}$here because $\mathrm{WTe}_{2}$ lacks the required in-plane mirror symmetry. One possible explanation in this case is a high spin-orbit scattering rate $\tau_{\text {so }}^{-1}$. Fitting the predicted form for $T_{\mathrm{c}}$ in a parallel field (29) to the data in the inset to Fig. $2 \mathrm{C}$ gives $\tau_{\mathrm{so}}^{-1} \approx 2 \mathrm{ps}^{-1}$ (23). Another possibility is that the Pauli limit is not actually exceeded, but that the effective g-factor in $\mathrm{WTe}_{2}$ is smaller than 2 owing to the strong spinorbit coupling.

The data in Fig. 2 display several other features worthy of mention. First, at intermediate magnetic fields the resistance approaches a $T$-independent level as $T \rightarrow 0$ that is orders of magnitude below the normal-state resistance. The data from Fig. 2B are replotted vs $1 / T$ in Fig. 2D to highlight the behavior below $100 \mathrm{mK}$. Similar behavior is seen at $B=$ 0 (Fig. 2A) for intermediate $n_{e}$, adding to the growing body of evidence that this is a robust phenomenon occurring in thin films close to superconductivity (30). Second, even at the lowest temperature, $R_{x x}$ rises smoothly from zero as a function of $B_{\perp}$ (Fig. 2E) whereas the onset of measurable resistance as a function of $B_{\|}$is relatively sudden, occurring above $2 \mathrm{~T}$ (Fig. 2F). Third, an intermediate plateau is visible in the $R_{x x}-T$ data at $B=0$ over a wide range of $n_{e}$ (Fig. 2A). It is extremely sensitive to $B_{\perp}$, almost disappearing at only 2 $\mathrm{mT}$ (Fig. 2B), whereas it survives in $B_{\|}$to above $2 \mathrm{~T}$ (Fig. $2 \mathrm{C}$ and inset to Fig. 2F). A similar feature has been reported in some other quasi-2D superconductors (31-33), but its nature, and the role of disorder, remain unresolved.

The high tunability of this $2 \mathrm{D}$ superconducting system invites comparison with theoretical predictions for critical behavior close to a quantum phase transition. Figure 3 shows how $R_{x x}$ depends on doping at a series of temperatures, along the dashed lines in the inset phase diagram. 
The $T$ dependence changes sign at $n_{\text {crit }} \approx 5 \times 10^{12} \mathrm{~cm}^{-2}$. In the inset we show an attempt to collapse the data onto a single function of $\left|1-n_{e} / n_{\text {crit }}\right| T^{-\alpha}$. The procedure is somewhat hindered by the fluctuations, which can be seen to be largely reproducible. The best-fit critical exponent $\alpha=0.8$ is similar to that reported for some insulator-superconductor transitions in thin films (34), although we note that the anomalous behavior near $n_{\text {crit }}$ mentioned above is not consistent with such a scaling.

Superconductivity induced by simple electrostatic gating in a monolayer of material that is not normally superconducting is intriguing, but perhaps even more interesting is that the ungated state is a $2 \mathrm{D}$ TI. This prompts the question of whether the helical edge channels remain when the superconductivity appears, and if so, how strongly they couple to it. In principle, $R_{x x}$ includes contributions from both edges and bulk. However, because in device M1 the edge conduction freezes out below $1 \mathrm{~K}$, in order to investigate the combination of edge channels and superconductivity we turn to another device, M2, in which edge conduction persists to lower temperatures (23).

Figure 4 shows measurements of the conductance, $G$, between adjacent contacts in $\mathrm{M} 2$ as a function of gate doping. The figure includes schematics indicating the inferred state of the edge (red for conducting), as well as the bulk state (colored to match the phase diagram). Consider first the black trace, taken at $200 \mathrm{mK}$ and $B_{\perp}=0$. At low $n_{e}$ the bulk is insulating and edge conduction dominates, albeit with large mesoscopic fluctuations. For $n_{e}>2 \times 10^{12} \mathrm{~cm}^{-2}, G$ increases as bulk conduction begins, then once $n_{e}$ exceeds $n_{\text {crit }}$ it increases faster as superconductivity appears, before leveling out at around $200 \mu \mathrm{S}$ owing to contact resistance. This interpretation is supported by warming to $1 \mathrm{~K}$ (red dotted trace), which destroys the superconductivity and so reduces $G$ for $n_{e}>n_{\text {crit, }}$, but enhances the edge conduction at low $n_{e}$ toward the ideal value of $e^{2} / h=39 \mu \mathrm{S}$. (We note that this $T$ dependence of the edge is associated with a gap of around $100 \mu \mathrm{eV}$, visible in the inset map of differential conductance vs bias and doping). A perpendicular field $B_{\perp}$ of 50 $\mathrm{mT}$ (green trace) also destroys the superconductivity, causing the conductance to fall for $n_{e}>n_{\text {crit }}$ but barely affecting it at lower $n_{e}$. High magnetic fields have been shown (9) to suppress edge conduction in the 2D TI state by breaking the time reversal symmetry. This effect can be clearly seen in the $B_{\perp}=1 \mathrm{~T}$ data (orange trace in Fig. 4 ) as $G$ falls to zero at low $n_{e}$. Importantly, comparing the green $\left(B_{\perp}=0.05 \mathrm{~T}\right)$ and orange $\left(B_{\perp}=1 \mathrm{~T}\right)$ traces, $G$ falls by a similar amount at higher $n_{e}$, consistent with a scenario in which the edge conduction supplies a parallel contribution, implying that helical edge states persist when $n_{e}>n_{\text {crit }}$ and at temperatures below $T_{\text {c. }}$.

This discovery raises compelling questions for future in- vestigation. It is likely that the helical edge modes persist when the superconductivity is restored by reducing the magnetic field to zero. Other techniques, such as scanning tunneling microscopy or contacts discriminating edge from bulk, may be needed to probe the edges separately from the bulk. The measurements presented here cannot determine the degree or nature of the coupling between superconductivity and edge conduction. One key question is whether the edge states also develop a superconducting gap, in which case they could host Majorana zero modes (5).

Another question concerns the nature of the superconducting order. It is striking that $n_{\text {crit }}$ corresponds to only $\sim 0.5 \%$ of an electron per $\mathrm{W}$ atom, which is about ten times lower than the doping level needed to observe superconductivity in other transition metal dichalcogenide monolayers (18). Many-layer $\mathrm{WTe}_{2}$ is semimetallic (35-38) under ambient conditions, with near-perfect compensation of electrons and holes, but becomes superconducting as the ratio of electrons to holes increases at high pressure (39). Some related materials, such as $\mathrm{TiSe}_{2}$, are known to switch from chargedensity-wave to superconducting states at quite low doping (40) or under pressure (41). We therefore speculate that doping tips the balance in monolayer $\mathrm{WTe}_{2}$ in favor of superconductivity, away from a competing insulating electronic ordering. Finally, given the topological band structure and likely strong correlations in this material, it is possible that the pairing is unconventional and perhaps topologically nontrivial.

\section{REFERENCES AND NOTES}

1. M. Z. Hasan, C. L. Kane, Colloquium: Topological insulators. Rev. Mod. Phys. 82 , 3045-3067 (2010). doi:10.1103/RevModPhys.82.3045

2. B. A. Bernevig, T. L. Hughes, S.-C. Zhang, Quantum spin Hall effect and topological phase transition in HgTe quantum wells. Science 314, 1757-1761 (2006). doi:10.1126/science. 1133734 Medline

3. C. L. Kane, E. J. Mele, Z2 topological order and the quantum spin Hall effect. Phys. Rev. Lett. 95, 146802 (2005). Medline

4. M. König, S. Wiedmann, C. Brüne, A. Roth, H. Buhmann, L. W. Molenkamp, X.-L. Qi, S.-C. Zhang, Quantum spin hall insulator state in $\mathrm{HgTe}$ quantum wells. Science 318, 766-770 (2007). doi:10.1126/science.1148047 Medline

5. L. Fu, C. L. Kane, Superconducting proximity effect and Majorana fermions at the surface of a topological insulator. Phys. Rev. Lett. 100, 096407 (2008). doi:10.1103/PhysRevLett.100.096407 Medline

6. S. Sarma, M. Freedman, C. Nayak, Majorana zero modes and topological quantum computation. NPJ Quantum Inform. 1, 15001 (2015). doi:10.1038/npjqi.2015.1

7. M. Sato, Y. Ando, Topological superconductors: A review. Rep. Prog. Phys. 80 , 076501 (2017). doi:10.1088/1361-6633/aa6ac7 Medline

8. X. Qian, J. Liu, L. Fu, J. Li, Quantum spin Hall effect in two-dimensional transition metal dichalcogenides. Science 346, 1344-1347 (2014). doi:10.1126/science.1256815 Medline

9. Z. Fei, T. Palomaki, S. Wu, W. Zhao, X. Cai, B. Sun, P. Nguyen, J. Finney, X. Xu, D. H. Cobden, Edge conduction in monolayer WTe2. Nat. Phys. 13, 677-682 (2017). doi:10.1038/nphys4091

10. S. Tang, C. Zhang, D. Wong, Z. Pedramrazi, H.-Z. Tsai, C. Jia, B. Moritz, M. 
Claassen, H. Ryu, S. Kahn, J. Jiang, H. Yan, M. Hashimoto, D. Lu, R. G. Moore, C.C. Hwang, C. Hwang, Z. Hussain, Y. Chen, M. M. Ugeda, Z. Liu, X. Xie, T. P. Devereaux, M. F. Crommie, S.-K. Mo, Z.-X. Shen, Quantum spin Hall state in monolayer $1 T^{\prime}-W T e_{2}$. Nat. Phys. 13, 683-687 (2017). doi:10.1038/nphys4174

11. L. Peng, Y. Yuan, G. Li, X. Yang, J.-J. Xian, C.-J. Yi, Y.-G. Shi, Y.-S. Fu, Observation of topological states residing at step edges of WTe2. Nat. Commun. 8, 659 (2017). doi:10.1038/s41467-017-00745-8 Medline

12. Z.-Y. Jia, Y.-H. Song, X.-B. Li, K. Ran, P. Lu, H.-J. Zheng, X.-Y. Zhu, Z.-Q. Shi, J. Sun, J. Wen, D. Xing, S.-C. Li, Direct visualization of a two-dimensional topological insulator in the single-layer 1T WTe2. Phys. Rev. B 96, 041108 (2017). doi:10.1103/PhysRevB.96.041108

13. S. Wu, V. Fatemi, Q. D. Gibson, K. Watanabe, T. Taniguchi, R. J. Cava, P. JarilloHerrero, Observation of the quantum spin Hall effect up to 100 kelvin in a monolayer crystal. Science 359, 76-79 (2018). doi:10.1126/science.aan6003 Medline

14. A. W. Tsen, B. Hunt, Y. D. Kim, Z. J. Yuan, S. Jia, R. J. Cava, J. Hone, P. Kim, C. R. Dean, A. N. Pasupathy, Nature of the quantum metal in a two-dimensional crystalline superconductor. Nat. Phys. 12, 208-212 (2015). doi:10.1038/nphys3579

15. X. Xi, Z. Wang, W. Zhao, J.-H. Park, K. T. Law, H. Berger, L. Forró, J. Shan, K. F. Mak, Ising pairing in superconducting $\mathrm{NbSe}_{2}$ atomic layers. Nat. Phys. 12, 139143 (2016). doi:10.1038/nphys3538

16. Q. Y. Wang et al., Interface-Induced High-Temperature Superconductivity in Single Unit-Cell FeSe Films on $\mathrm{SrTiO}_{3}$. Chin. Phys. Lett. 29, 4 (2012).

17. L. J. Li, E. C. T. O'Farrell, K. P. Loh, G. Eda, B. Özyilmaz, A. H. Castro Neto, Controlling many-body states by the electric-field effect in a two-dimensional material. Nature 529, 185-189 (2016). doi:10.1038/nature16175 Medline

18. Y. Fu, E. Liu, H. Yuan, P. Tang, B. Lian, G. Xu, J. Zeng, Z. Chen, Y. Wang, W. Zhou, K. Xu, A. Gao, C. Pan, M. Wang, B. Wang, S.-C. Zhang, Y. Cui, H. Y. Hwang, F. Miao, Gated tuned superconductivity and phonon softening in monolayer and bilayer MoS2. NPJ Quantum Mater. 2, 52 (2017). doi:10.1038/s41535-017-0056-1

19. Y. Saito, T. Nojima, Y. Iwasa, Gate-induced superconductivity in two-dimensional atomic crystals. Supercond. Sci. Technol. 29, 093001 (2016). doi:10.1088/09532048/29/9/093001

20. K. Ueno, S. Nakamura, H. Shimotani, A. Ohtomo, N. Kimura, T. Nojima, H. Aoki, Y. Iwasa, M. Kawasaki, Electric-field-induced superconductivity in an insulator. Nat. Mater. 7, 855-858 (2008). doi:10.1038/nmat2298 Medline

21. J. M. Lu, O. Zheliuk, I. Leermakers, N. F. Q. Yuan, U. Zeitler, K. T. Law, J. T. Ye, Evidence for two-dimensional Ising superconductivity in gated MoS 2 . Science 350, 1353-1357 (2015). doi:10.1126/science.aab2277 Medline

22. D. Costanzo, S. Jo, H. Berger, A. F. Morpurgo, Gate-induced superconductivity in atomically thin MoS 2 crystals. Nat. Nanotechnol. 11, 339-344 (2016). doi:10.1038/nnano.2015.314 Medline

23. See supplementary materials.

24. R. A. Klemm, Pristine and intercalated transition metal dichalcogenide superconductors. Physica C 514, 86-94 (2015). doi:10.1016/j.physc.2015.02.023

25. M. Tinkham, Introduction to Superconductivity (McGraw Hill, ed. 2, 1996).

26. Y. Saito, T. Nojima, Y. Iwasa, Highly crystalline 2D superconductors. Nat. Rev. Mater. 2, 16094 (2016). doi:10.1038/natrevmats.2016.94

27. H. Y. Lv, W. J. Lu, D. F. Shao, Y. Liu, S. G. Tan, Y. P. Sun, Perfect charge compensation in $\mathrm{WTe}_{2}$ for the extraordinary magnetoresistance: From bulk to monolayer. Europhys. Lett. 110, 37004 (2015). doi:10.1209/02955075/110/37004

28. A. M. Clogston, Upper limit for critical field in hard superconductors. Phys. Rev. Lett. 9, 266-267 (1962). doi:10.1103/PhysRevLett.9.266

29. R. A. Klemm, A. Luther, M. R. Beasley, Theory of the upper critical field in layered superconductors. Phys. Rev. B 12, 877-891 (1975). doi:10.1103/PhysRevB.12.877
30. A. Kapitulnik, S. A. Kivelson, B. Spivak, Anomalous metals-failed superconductors. arXiv:1712.07215 (2017).

31. Y. Cheng, M. B. Stearns, Superconductivity of $\mathrm{Nb} / \mathrm{Cr}$ multilayers. J. Appl. Phys. 67, 5038-5040 (1990). doi:10.1063/1.344716

32. L. Z. Deng, B. Lv, K. Zhao, F. Y. Wei, Y. Y. Xue, Z. Wu, C. W. Chu, Evidence for defect-induced superconductivity up to $49 \mathrm{~K}$ in $\left(\mathrm{Ca}_{1-x} \mathrm{R} \mathrm{R}\right) \mathrm{Fe}$-As2. Phys. Rev. B 93 , 054513 (2016). doi:10.1103/PhysRevB.93.054513

33. S.-G. Jung, S. Shin, H. Jang, P. Mikheenko, T. H. Johansen, T. Park, Effects of magnetic impurities on upper critical fields in the high- $\mathrm{T}_{\mathrm{c}}$ superconductor Ladoped $\mathrm{CaFe}_{2} \mathrm{As}_{2}$. Supercond. Sci. Technol. 30, 085009 (2017). doi:10.1088/1361-6668/aa766c

34. A. M. Goldman, Superconductor-insulator transitions. Int. J. Mod. Phys. B 24 , 4081-4101 (2010). doi:10.1142/S0217979210056451

35. A. A. Soluyanov, D. Gresch, Z. Wang, Q. Wu, M. Troyer, X. Dai, B. A. Bernevig, Type-II Weyl semimetals. Nature 527, 495-498 (2015). doi:10.1038/nature15768 Medline

36. M. N. Ali, J. Xiong, S. Flynn, J. Tao, Q. D. Gibson, L. M. Schoop, T. Liang, N. Haldolaarachchige, M. Hirschberger, N. P. Ong, R. J. Cava, Large, non-saturating magnetoresistance in WTe2. Nature 514, 205-208 (2014). doi:10.1038/nature13763 Medline

37. D. MacNeill, G. M. Stiehl, M. H. D. Guimaraes, R. A. Buhrman, J. Park, D. C. Ralph, Control of spin-orbit torques through crystal symmetry in $\mathrm{WTe}_{2} /$ ferromagnet bilayers. Nat. Phys. 13, 300-305 (2017). doi:10.1038/nphys3933

38. V. Fatemi, Q. D. Gibson, K. Watanabe, T. Taniguchi, R. J. Cava, P. Jarillo-Herrero, Magnetoresistance and quantum oscillations of an electrostatically tuned semimetal-to-metal transition in ultrathin WTe2. Phys. Rev. B 95, 041410 (2017). doi:10.1103/PhysRevB.95.041410

39. D. Kang, Y. Zhou, W. Yi, C. Yang, J. Guo, Y. Shi, S. Zhang, Z. Wang, C. Zhang, S. Jiang, A. Li, K. Yang, Q. Wu, G. Zhang, L. Sun, Z. Zhao, Superconductivity emerging from a suppressed large magnetoresistant state in tungsten ditelluride. Nat. Commun. 6, 7804 (2015). doi:10.1038/ncomms8804 Medline

40. E. Morosan, H. W. Zandbergen, B. S. Dennis, J. W. G. Bos, Y. Onose, T. Klimczuk, A. P. Ramirez, N. P. Ong, R. J. Cava, Superconductivity in $\mathrm{Cu}_{x} \mathrm{TiSe}$. Nat. Phys. 2 , 544-550 (2006). doi:10.1038/nphys360

41. A. F. Kusmartseva, B. Sipos, H. Berger, L. Forró, E. Tutiš, Pressure induced superconductivity in pristine 1T-TiSe 2. Phys. Rev. Lett. 103, 236401 (2009). doi:10.1103/PhysRevLett.103.236401 Medline

42. P. J. Zomer, M. H. D. Guimaraes, J. C. Brant, N. Tombros, B. J. van Wees, Fast pick up technique for high quality heterostructures of bilayer graphene and hexagonal boron nitride. Appl. Phys. Lett. 105, 013101 (2014). doi:10.1063/1.4886096

43. K. Maki, Effect of Pauli Paramagnetism on Magnetic Properties of High-Field Superconductors. Phys. Rev. 148, 362-369 (1966). doi:10.1103/PhysRev.148.362

\section{ACKNOWLEDGMENTS}

We thank Oded Agam, Anton Andreev and Boris Spivak for discussions, and Jiaqiang Yan for the WTe 2 crystals. Funding: TP, YF, WZ, XX, and DC supported by the U.S. Department of Energy, Office of Basic Energy Sciences, Division of Materials Sciences and Engineering, Awards DE-SC0002197 (DHC) and DE-SC0018171 (XX); AFOSR FA9550-14-1-0277; NSF EFRI 2DARE 1433496; and NSF MRSEC 1719797. ES, PB, C0, SL and JF supported by the Canada Foundation for Innovation; the National Science and Engineering Research Council; CIFAR; and SBQMI. Author contributions: TP, $X X$, and DC conceived of the original experiment; TP, ZF, and WZ fabricated the devices; $E S, Z F, P B$, and $\mathrm{CO}$ carried out the measurements under the primary supervision of JF and DC in a cryostat developed by ES, SL, and JF; all authors contributed to the analysis of the data; ES, JF, and DC wrote the manuscript; all authors contributed to the final editing of the manuscript. Competing interests: The authors declare no competing interests. Data and materials availability: The data shown in the paper are available at https://github.com/EbrahimSajadi/2D_SC_TI. 


\section{SUPPLEMENTARY MATERIALS}

www.sciencemag.org/cgi/content/full/science.aar4426/DC1

Materials and Methods

Supplementary Text

Figs. S1 to S10

Table S1

References $(42,43)$

12 November 2017; accepted 5 October 2018

Published online 25 October 2018

10.1126/science.aar4426 

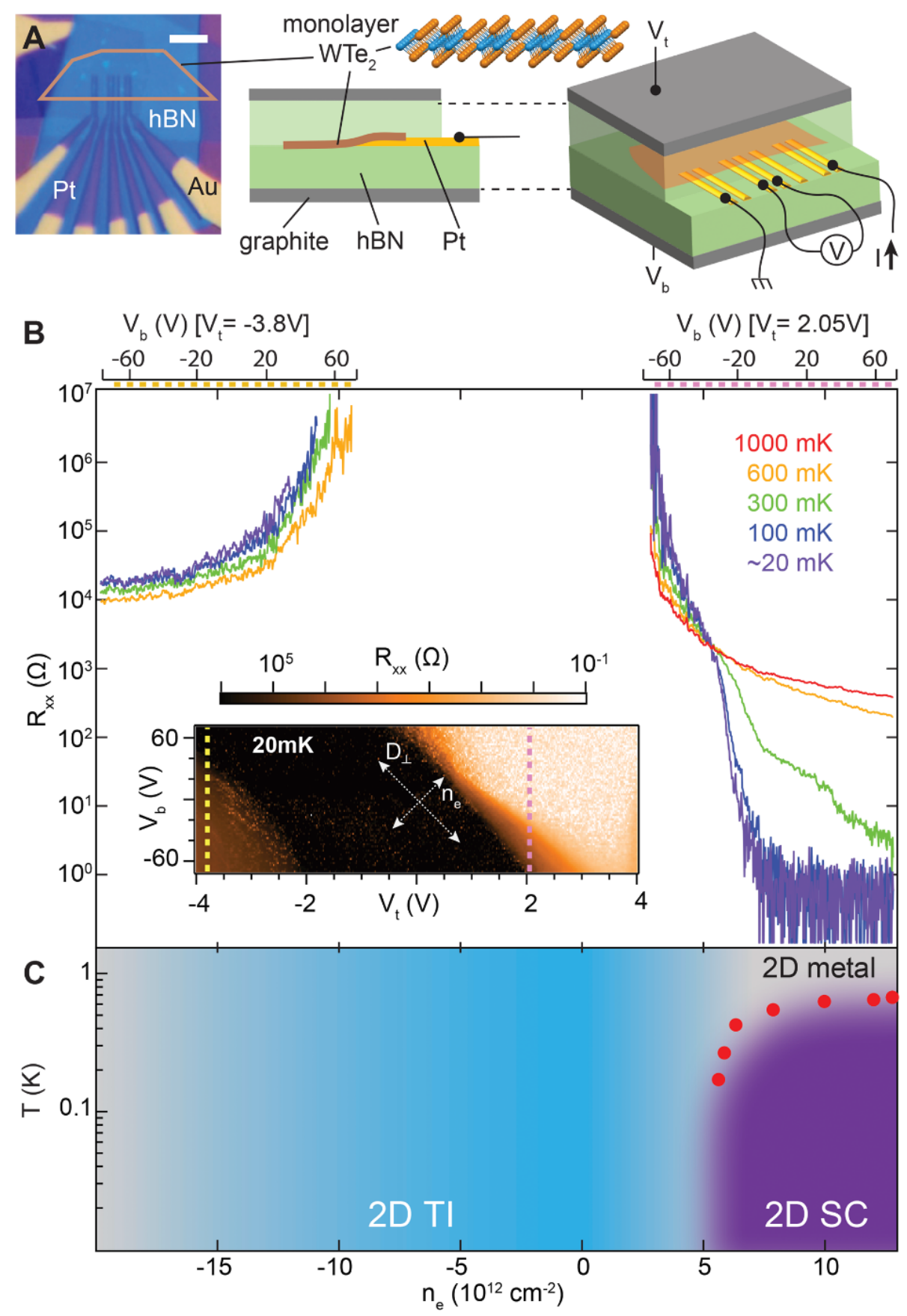

Fig. 1. Characteristics of monolayer $\mathrm{WTe}_{2}$ device $\mathrm{M} 1$ at temperatures below $1 \mathrm{~K}$. (A) Optical image (the white scale bar indicates $5 \mu \mathrm{m}$ ) and schematic device structure, showing current, voltage contacts and ground configuration for measuring the 4-probe resistance $R_{x x}$. Inset: schematic of the atomic structure of monolayer WTe $e_{2}$. (B) $R_{x x}$ as a function of electrostatic doping $\left(n_{e}\right)$ at a series of temperatures. Inset: variation of $R_{x x}$ at $20 \mathrm{mK}$ with top and bottom gate voltages, $V_{\mathrm{t}}$ and $V_{\mathrm{b}}$, indicating the axes corresponding to doping $n_{e}$ and transverse displacement field $D_{\perp}$. $R_{x x}$ depends primarily on $n_{e}$ and only weakly on $D_{\perp}$. The measurements in the main panel for $n_{e}>0$ and $n_{e}<0$ were made separately, sweeping $V_{\mathrm{b}}$ along the two colored dashed lines in the inset to avoid contact effects. (C) Phase diagram constructed from measurements in this paper. 

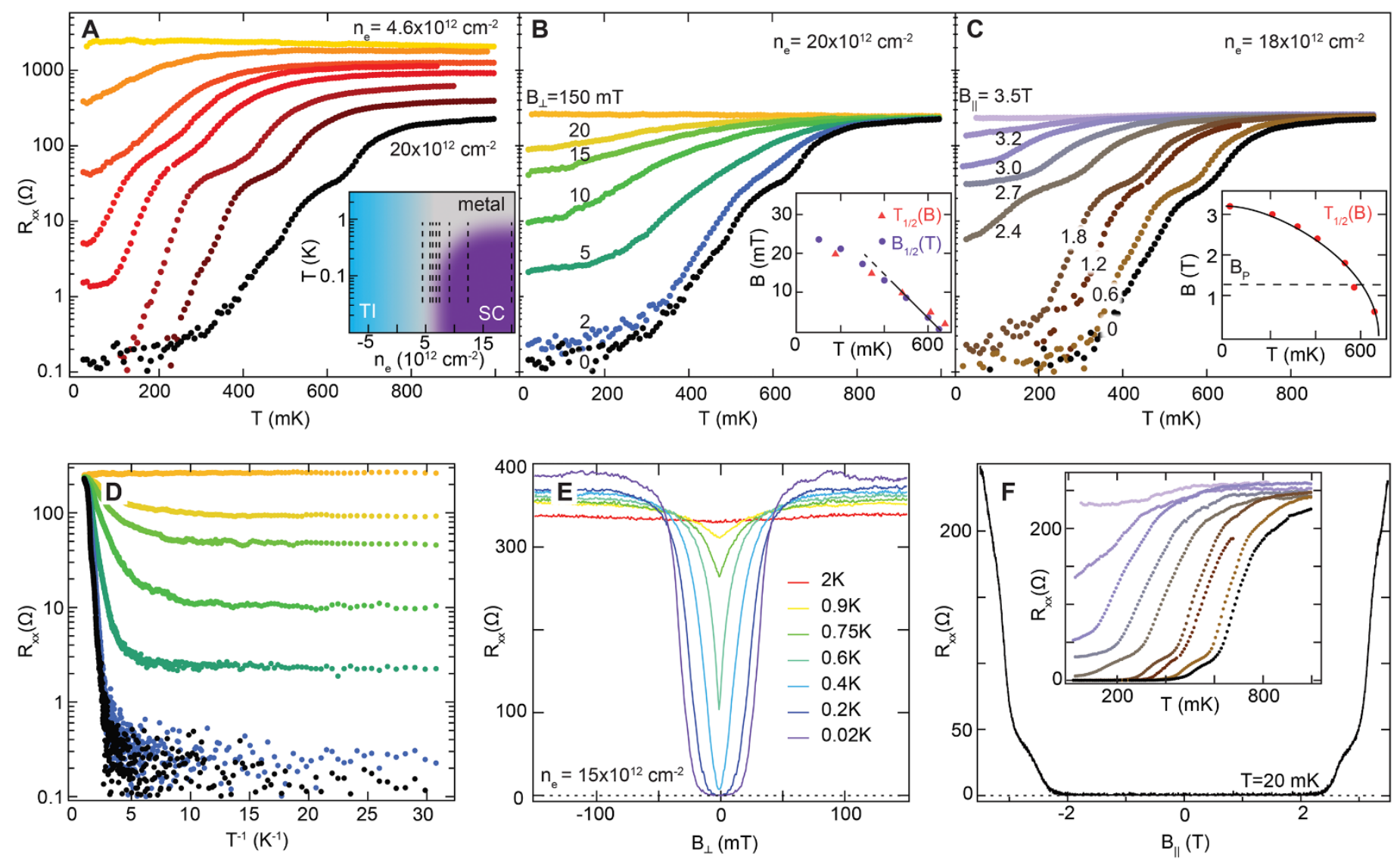

Fig. 2. Resistance characterization of device $M 1$ in the superconducting regime. (A) $R_{x x}$ on log scale vs temperature $T$ at a series of positive-gate doping levels $n_{e}\left[20,12,8.5,6.7,6.1,5.6,5,4.6 \times 10^{12} \mathrm{~cm}^{-2}\right]$ showing a drop of several orders of magnitude at low $T$ for larger $n_{e}$. Inset: location of sweeps on the phase diagram. (B) Effect of perpendicular magnetic field, $B_{\perp}$ on resistance at the highest $n_{e}$ in $(A)$. (Demagnetization effects are neglected in light of the finite resistivity of the sample.) Inset: characteristic temperatures $T_{1 / 2}$ obtained from these temperature sweeps, as well as characteristic fields $B_{1 / 2}$ measured from field sweeps under similar conditions. (C) Same as (B) but for in-plane magnetic field, $B_{\|}$. $\left(B_{\|}=0\right.$ data are for $n_{e}=19 \times 10^{12} \mathrm{~cm}^{-2}$ and the rest of the data are for $n_{e}=18 \times 10^{12}$ ). Inset shows reduction of $T_{1 / 2}$ with $B_{\|}$, fit to the expected form for materials with strong spin-orbit scattering (solid line). The Pauli limit $B_{\mathrm{P}}$, assuming $g=2$, is indicated by the dashed line. (D) Data from (B) replotted to highlight the saturation of $R_{x x}$ at low $T$. (E) Sweeps of $B_{\perp}$ showing rise of resistance beginning at very low field. (F) Sweep of $B_{\|}$showing sharper onset of resistance compared to (E). Inset: data from (C) on a linear scale. 


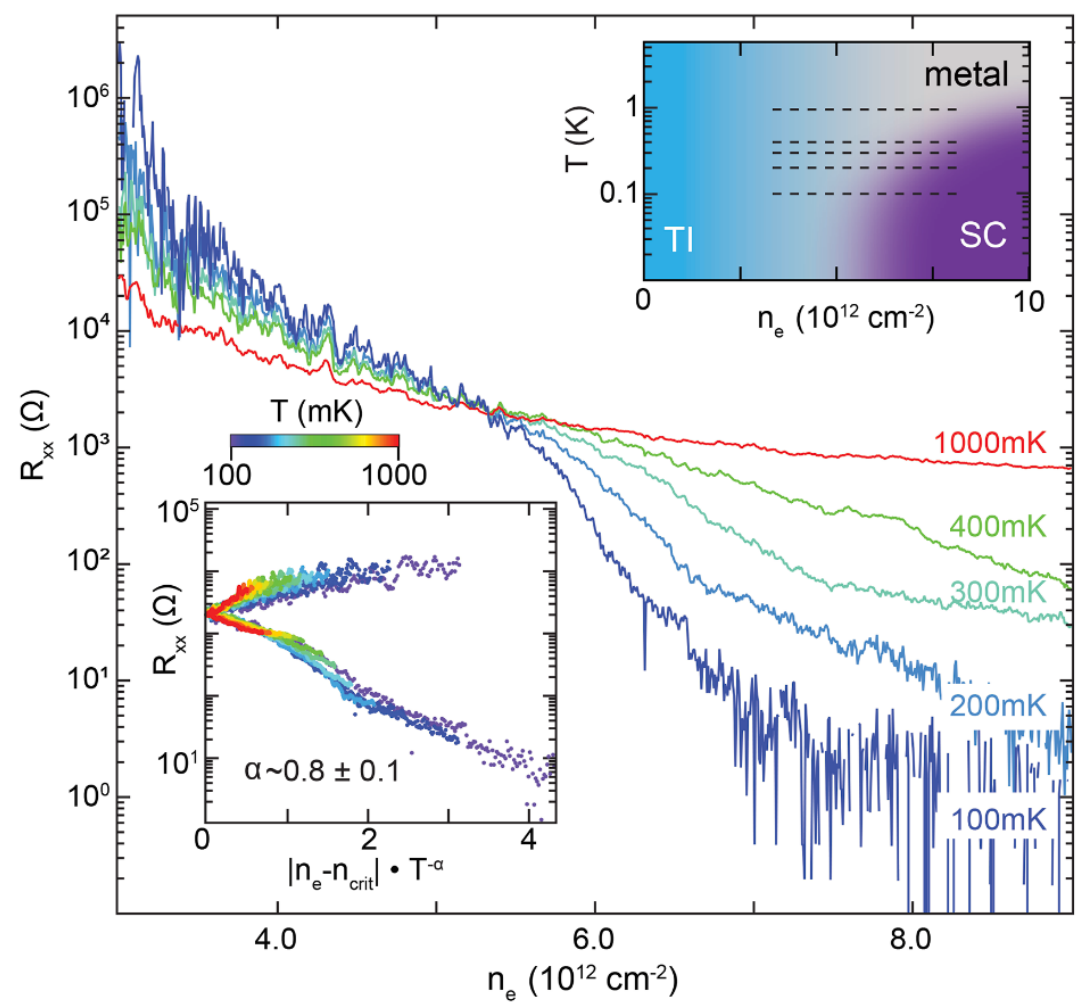

Fig. 3. Scaling analysis of the transition. Main panel: Multiple $R_{x x}$ vs doping traces, taken at different temperatures, cross at a critical doping level $n_{\text {crit }} \approx 5 \times 10^{12} \mathrm{~cm}^{-2}$. Upper inset: dashed lines locate these sweeps on the phase diagram. Lower inset: same data presented on a scaling plot, taking critical exponent $\alpha=0.8$. 


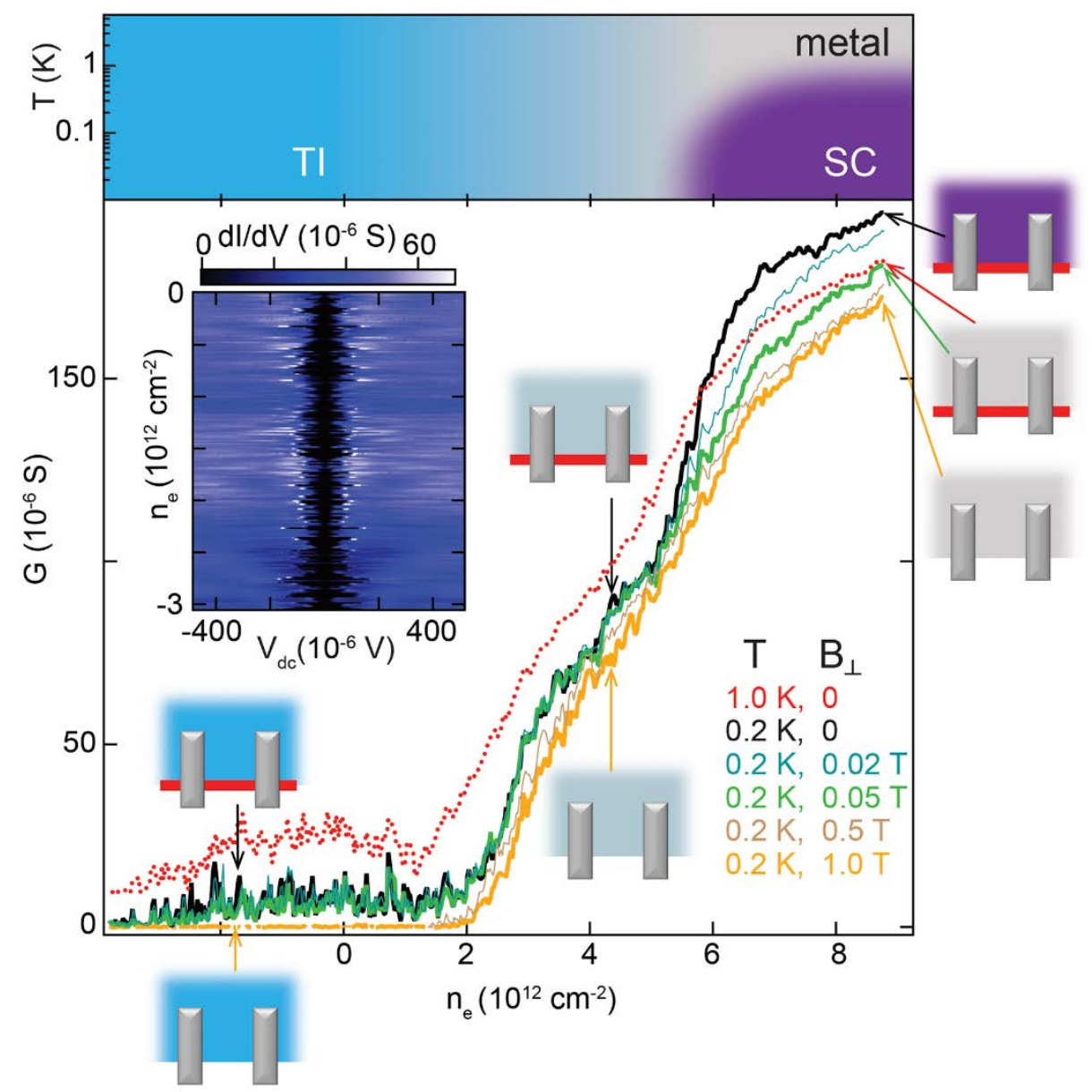

Fig. 4. Evidence for the presence of both edge conduction and superconductivity in device M2. The main panel shows the linear conductance between two adjacent contacts vs gate doping at the temperatures and perpendicular magnetic fields noted. Schematics indicate the state of edge and bulk conduction at different points, the bulk being colored to match the phase diagram reproduced above, and red indicating a conducting edge state. Superconductivity occurs for $n_{e}>5 \times 10^{12} \mathrm{~cm}^{-2}$ at $B=0$; the zero resistance state, disguised by contact resistance in this figure, was confirmed in a separate 4wire measurement of R vs T (fig. S10); edge conduction dominates for $n_{e}<2 \times 10^{12} \mathrm{~cm}^{-2}$ but appears to be present at all $n_{e}$. Inset: color-scale plot of differential conductance vs dc voltage bias and doping level, revealing a gap of around $100 \mu \mathrm{eV}$ that fluctuates rapidly as a function of doping level. 